\title{
Effects of Combination Lipid Therapy in Type 2 Diabetes Mellitus
}

\author{
The ACCORD Study Group*
}

\begin{abstract}
BACKGROUND-We investigated whether combination therapy with a statin plus a fibrate, as compared with statin monotherapy, would reduce the risk of cardiovascular disease in patients with type 2 diabetes mellitus who were at high risk for cardiovascular disease.

METHODS-We randomly assigned 5518 patients with type 2 diabetes who were being treated with open-label simvastatin to receive either masked fenofibrate or placebo. The primary outcome was the first occurrence of nonfatal myocardial infarction, nonfatal stroke, or death from cardiovascular causes. The mean follow-up was 4.7 years.
\end{abstract}

\begin{abstract}
RESULTS-The annual rate of the primary outcome was $2.2 \%$ in the fenofibrate group and $2.4 \%$ in the placebo group (hazard ratio in the fenofibrate group, $0.92 ; 95 \%$ confidence interval [CI], 0.79 to $1.08 ; \mathrm{P}=0.32$ ). There were also no significant differences between the two study groups with respect to any secondary outcome. Annual rates of death were $1.5 \%$ in the fenofibrate group and $1.6 \%$ in the placebo group (hazard ratio, $0.91 ; 95 \% \mathrm{CI}, 0.75$ to $1.10 ; \mathrm{P}=0.33$ ). Prespecified subgroup analyses suggested heterogeneity in treatment effect according to sex, with a benefit for men and possible harm for women $(\mathrm{P}=0.01$ for interaction), and a possible interaction according to lipid subgroup, with a possible benefit for patients with both a high baseline triglyceride level and a low baseline level of high-density lipoprotein cholesterol ( $\mathrm{P}=0.057$ for interaction).
\end{abstract}

CONCLUSIONS-The combination of fenofibrate and simvastatin did not reduce the rate of fatal cardiovascular events, nonfatal myocardial infarction, or nonfatal stroke, as compared with simvastatin alone. These results do not support the routine use of combination therapy with fenofibrate and simvastatin to reduce cardiovascular risk in the majority of high-risk patients with type 2 diabetes. (ClinicalTrials.gov number, NCT00000620.)

Patients with type 2 diabetes mellitus have an increased incidence of atherosclerotic cardiovascular disease $.1^{-} 4$ This increase is attributable, in part, to associated risk factors, including hypertension and dyslipidemia. The latter is characterized by elevated plasma triglyceride levels, low levels of high-density lipoprotein (HDL) cholesterol, and small, dense low-density lipoprotein (LDL) particles.5,6 The Action to Control Cardiovascular Risk in Diabetes (ACCORD) study was designed to test the effect of intensive treatment of blood glucose and either blood pressure or plasma lipids on cardiovascular outcomes in 10,251

\footnotetext{
Copyright $\odot 2010$ Massachusetts Medical Society.

Address reprint requests to Dr. Ginsberg at the Department of Medicine, Columbia University College of Physicians and Surgeons, Rm. PH 10-305, New York, NY 10032, or at hng1@ columbia.edu.

*The members of the Action to Control Cardiovascular Risk in Diabetes (ACCORD) Study Group are listed in Section 20 in

Supplementary Appendix 1, available with the full text of this article at NEJM.org. The affiliations of members of the Writing Committee are listed in the Appendix.

The members of the Writing Committee (Henry N. Ginsberg, M.D., Marshall B. Elam, M.D., Laura C. Lovato, M.S., John R. Crouse III, M.D., Lawrence A. Leiter, M.D., Peter Linz, M.D., William T. Friedewald, M.D., John B. Buse, M.D., Ph.D., Hertzel C. Gerstein, M.D., Jeffrey Probstfield, M.D., Richard H. Grimm, M.D., Ph.D., Faramarz Ismail-Beigi, M.D., Ph.D., J. Thomas Bigger, M.D., David C. Goff, Jr., M.D., Ph.D., William C. Cushman, M.D., Denise G. Simons-Morton, M.D., Ph.D., and Robert P. Byington, Ph.D.) assume responsibility for the integrity of the article.

Disclosure forms provided by the authors are available with the full text of this article at NEJM.org.
} 
patients with type 2 diabetes who were at high risk for cardiovascular disease. Here we present the findings of the ACCORD lipid trial (ACCORD Lipid).

Although statins are efficacious in patients with type 2 diabetes, rates of cardiovascular events remain elevated in such patients even after statin treatment. ${ }^{7-9}$ Fibrate therapy in patients with type 2 diabetes reduced the rate of coronary heart disease events in the Veterans Affairs HDL Intervention Trial (VA-HIT; ClinicalTrials.gov number, NCT00035711) ${ }^{10}$ but not in the Fenofibrate Intervention and Event Lowering in Diabetes (FIELD) trial (Current Controlled Trials number, ISRCTN64783481). ${ }^{11}$ However, a post hoc analysis of data from the FIELD study suggested a benefit for patients with both elevated triglyceride levels and low HDL cholesterol levels.12 Previous fibrate studies in subjects with diabetes10,11 or in those without diabetes $13^{-15}$ did not address the role of such drugs in patients receiving statin therapy. The hypothesis that we tested in ACCORD Lipid was that in high-risk patients with type 2 diabetes, combination treatment with a fibrate (both to raise HDL cholesterol levels and to lower triglyceride levels) and a statin (to reduce LDL cholesterol levels) would reduce the rate of cardiovascular events, as compared with treatment with a statin alone.

\section{METHODS STUDY DESIGN}

The rationale and designs for the various components of ACCORD have been reported previously. ${ }^{16-20}$ The ACCORD study was a randomized trial conducted at 77 clinical sites organized into seven networks in the United States and Canada. (For a full list of participating institutions and investigators, see Section 20 in Supplementary Appendix 1, available with the full text of this article at NEJM.org The trial was sponsored by the National Heart, Lung, and Blood Institute (NHLBI), and the protocol was approved by a review panel at the NHLBI, as well as by the institutional review board or ethics committee at each center.

In the ACCORD study, all patients were randomly assigned to receive either intensive glycemic control (targeting a glycated hemoglobin level below 6.0\%) or standard therapy (targeting a glycated hemoglobin level of 7.0 to $7.9 \%$ ). The results of this comparison have been reported previously. ${ }^{20}$ A subgroup of patients in the ACCORD study were also enrolled in the ACCORD Lipid trial and underwent randomization, in a 2-by-2 factorial design, to receive simvastatin plus either fenofibrate or placebo. Randomization occurred between January 11, 2001, and October 29, 2005. End-of-study visits were scheduled between March and June 2009. Additional details regarding the trial protocol and amendments are provided in Supplementary Appendix 2, also available with the full text of this article at NEJM.org.

\section{ELIGIBILITY}

All patients in the ACCORD study had type 2 diabetes and a glycated hemoglobin level of $7.5 \%$ or more. If patients had evidence of clinical cardiovascular disease, the age range was limited to 40 to 79 years; if they had evidence of subclinical cardiovascular disease or at least two additional cardiovascular risk factors, the age range was compressed to 55 to 79 years. Patients were specifically eligible to participate in the lipid trial if they also had the following: an LDL cholesterol level of 60 to $180 \mathrm{mg}$ per deciliter (1.55 to $4.65 \mathrm{mmol}$ per liter), an HDL cholesterol level below $55 \mathrm{mg}$ per deciliter ( $1.42 \mathrm{mmol}$ per liter) for women and blacks or below $50 \mathrm{mg}$ per deciliter $(1.29 \mathrm{mmol}$ per liter) for all other groups, and a triglyceride level below $750 \mathrm{mg}$ per deciliter $(8.5 \mathrm{mmol}$ per liter $)$ if they were not receiving lipid therapy or below $400 \mathrm{mg}$ per deciliter $(4.5 \mathrm{mmol}$ per liter) if they were receiving lipid therapy. All patients provided written informed consent. Additional details regarding eligibility and the protocol for the enrollment of patients are available in Section 3 in Supplementary Appendix 1. 


\section{STUDY PROCEDURES}

Randomization was performed centrally on the trial's Web site with the use of permuted blocks to maintain concealment of study-group assignments. Open-label simvastatin therapy began at the randomization visit, and the masked administration of either fenofibrate or placebo began 1 month later. The initial dose of simvastatin complied with national lipid guidelines at the time the study began. ${ }^{21}$ The dose of simvastatin was modified over time in response to changing guidelines (see Section 6 in Supplementary Appendix 1). ${ }^{18}$

At the start of the trial, the dose of fenofibrate was $160 \mathrm{mg}$ per day. Because of a rise in serum creatinine levels in some patients while receiving this dose of fenofibrate, ${ }^{22}$ starting in 2004, the dose of fenofibrate was adjusted according to the estimated glomerular filtration rate (GFR) with the use of the abbreviated Modification of Diet in Renal Disease (MDRD) equation (see Section 7 in Supplementary Appendix 1). ${ }^{23}$

A fasting plasma lipid profile was measured at the ACCORD central laboratory at 4, 8, and 12 months after randomization, annually thereafter, and at the end of the study. Safety profiles, including liver-function tests and measurements of creatine kinase levels, were determined at $1,4,8$, and 12 months after randomization and annually thereafter. If symptoms or signs suggestive of drug-induced toxic effects developed, tests of liver function (including measurement of alanine aminotransferase), creatine kinase, or both were obtained. If liverfunction values were elevated, lipid medications were temporarily discontinued; if creatine kinase values were elevated, lipid medications were permanently discontinued.

\section{PRESPECIFIED OUTCOMES}

The prespecified primary outcome was the first occurrence of a major cardiovascular event, including nonfatal myocardial infarction, nonfatal stroke, or death from cardiovascular causes. Secondary outcomes included the combination of the primary outcome plus revascularization or hospitalization for congestive heart failure (termed the "expanded macrovascular outcome"); a combination of a fatal coronary event, nonfatal myocardial infarction, or unstable angina (termed "major coronary disease events"); nonfatal myocardial infarction; fatal or nonfatal stroke; nonfatal stroke; death from any cause; death from cardiovascular causes; and hospitalization or death due to heart failure. Definitions of each prespecified outcome and methods of ascertainment are detailed in Section 8 in Supplementary Appendix 1.

\section{STUDY OVERSIGHT}

Fenofibrate and matching placebo were donated by Abbott Laboratories; simvastatin was donated by Merck. The drug manufacturers had no role in the design of the study, in the accrual or analysis of the data, or in the preparation of the manuscript. All authors vouch for the accuracy and completeness of the reported data.

\section{STATISTICAL ANALYSIS}

The study was designed to recruit 5800 patients, with a power of $87 \%$ to detect a $20 \%$ reduction in the rate of the primary outcome for patients in the fenofibrate group, as compared with placebo, assuming a two-sided alpha level of 0.05 , a primary outcome rate of $2.4 \%$ per year in the placebo group, and an average follow-up of approximately 5.6 years for patients who did not have an event. All statistical analyses were conducted at the coordinating center with the use of S-Plus software, version 8.0 (Insightful) or SAS software, version 9.1 (SAS Institute). Baseline characteristics were compared between study groups with the use of the chi-square test, Fisher's exact test, Wilcoxon rank-sum test, and two-sample t-tests. The incidence of key safety outcomes was compared with the use of Fisher's exact test. 
Analyses of primary and secondary outcomes were performed with the use of time-to-event methods, according to the intention-to-treat principle, and occurrences of outcomes were compared with the use of hazard ratios and $95 \%$ confidence intervals. Two-sided $\mathrm{P}$ values were obtained from likelihood ratio tests from Cox proportional-hazards regression analyses. The Cox models contained a term representing study-group assignment plus terms for the following prespecified variables: assignment to the intensive glycemic intervention, the seven clinicalcenter networks, and the presence or absence of a previous cardiovascular event. Betweengroup differences were also examined in prespecified subgroups on 10 baseline characteristics (see Section 9 in Supplementary Appendix 1). Event rates are expressed as the percentage of events per years of follow-up, taking into account the censoring of follow-up data. KaplanMeier estimates were used to obtain the proportion of patients who had an event during followup.

The primary outcome and total rates of death were monitored by the data and safety monitoring board, using O'Brien-Fleming boundaries determined by the Lan-DeMets approach. For the primary outcome and rates of death, $\mathrm{P}$ values have been adjusted to account for the number, timing, and results of interim analyses. Further details regarding the analytic methods are available in Section 11 in Supplementary Appendix 1.

\section{RESULTS STUDY PATIENTS}

A total of 5518 patients were enrolled in the ACCORD Lipid study, with 2765 assigned to receive fenofibrate plus simvastatin and 2753 assigned to receive placebo plus simvastatin. Baseline characteristics were similar between the two groups (Table 1). The mean age was 62 years, and $31 \%$ of the patients were female. Thirty-seven percent had a history of a cardiovascular event, and about $60 \%$ were taking a statin before enrollment.

The mean duration of follow-up was 4.7 years for the primary outcome and 5.0 years for total rates of death. At the final study visit, $77.3 \%$ of the patients in the fenofibrate group and $81.3 \%$ of those in the placebo group were taking their assigned medication. At the end of the study, approximately $80 \%$ of patients were still taking simvastatin in each group, and an additional $6 \%$ were taking an alternative study-approved agent for lowering LDL cholesterol. Additional details related to adherence are in presented in Section 12 in Supplementary Appendix 1. The average daily dose of simvastatin during the follow-up period was $22.3 \mathrm{mg}$ in the fenofibrate group and $22.4 \mathrm{mg}$ in the placebo group.

\section{SAFETY}

Elevations of creatine kinase of more than 10 times the upper limit of the normal range at any time during the trial occurred in 10 patients $(0.4 \%)$ in the fenofibrate group and $9(0.3 \%)$ in the placebo group (for details, see Section 13 in Supplementary Appendix 1). An elevation in alanine aminotransferase of more than three times the upper limit of the normal range occurred in 52 patients $(1.9 \%)$ in the fenofibrate group and $40(1.5 \%)$ in the placebo group.

As noted in other fenofibrate trials, 11,22 mean serum creatinine levels increased from 0.93 to $1.10 \mathrm{mg}$ per deciliter ( 82 to $97 \mu \mathrm{mol}$ per liter) in the fenofibrate group within the first year and remained relatively stable thereafter. In the placebo group, mean serum creatinine levels increased from 0.93 to $1.04 \mathrm{mg}$ per deciliter ( 82 to $92 \mu \mathrm{mol}$ per liter) during the course of the trial (see Section 15 in Supplementary Appendix 1). The study drug was discontinued by 66 patients $(2.4 \%)$ in the fenofibrate group and $30(1.1 \%)$ in the placebo group because of a decrease in the estimated GFR. At the last clinic visit, 440 patients (15.9\%) in the fenofibrate group and $194(7.0 \%)$ in the placebo group were receiving a reduced dose of either fibrate or 
placebo because of a decreased estimated GFR. There was no significant between-group difference in the incidence of both hemodialysis and end-stage renal disease (75 patients in the fenofibrate group vs. 77 in the placebo group). There was a lower incidence of both microalbuminuria and macroalbuminuria in the fenofibrate group than in the placebo group (see Section 13 in Supplementary Appendix 1).

\section{PLASMA LIPIDS}

By the end of the study, the mean LDL cholesterol level fell from 100.0 to $81.1 \mathrm{mg}$ per deciliter ( 2.59 to $2.10 \mathrm{mmol}$ per liter) in the fenofibrate group and from 101.1 to $80.0 \mathrm{mg}$ per deciliter (2.61 to $2.07 \mathrm{mmol}$ per liter) in the placebo group (Fig. 1, and Section 16 in Supplementary Appendix 1). Mean HDL cholesterol levels increased from 38.0 to $41.2 \mathrm{mg}$ per deciliter ( 0.98 to $1.07 \mathrm{mmol}$ per liter) in the fenofibrate group and from 38.2 to $40.5 \mathrm{mg}$ per deciliter ( 0.99 to $1.05 \mathrm{mmol}$ per liter) in the placebo group. Median plasma triglyceride levels decreased from 164 to $122 \mathrm{mg}$ per deciliter (1.85 to $1.38 \mathrm{mmol}$ per liter) in the fenofibrate group and from 160 to $144 \mathrm{mg}$ per deciliter (1.81 to $1.63 \mathrm{mmol}$ per liter) in the placebo group.

\section{CLINICAL OUTCOMES}

The annual rate of the primary outcome was $2.2 \%$ in the fenofibrate group, as compared with $2.4 \%$ in the placebo group (hazard ratio in the fenofibrate group, $0.92 ; 95 \%$ confidence interval [CI], 0.79 to $1.08 ; \mathrm{P}=0.32$ after adjustment for monitoring) (Table 2 and Fig. 2). Hazard ratios for the secondary outcomes, including the individual components of the primary outcome, ranged from 0.82 to 1.17 ( $\mathrm{P} \geq 0.10$ for all comparisons) (Table 2). Annual rates of death from all causes were $1.5 \%$ in the fenofibrate group and $1.6 \%$ in the placebo group (hazard ratio, $0.91 ; 95 \%$ CI, 0.75 to $1.10 ; \mathrm{P}=0.33$ for the adjusted comparison). Specific causes of death and enlarged versions of the Figure 2 insets are presented in Sections 17 and 18 in Supplementary Appendix 1.

Study-group effects on the primary outcome across prespecified baseline subgroups are shown in Figure 3. Only sex showed evidence of an interaction according to study group: the primary outcome for men was $11.2 \%$ in the fenofibrate group versus $13.3 \%$ in the placebo group, whereas the rate for women was $9.1 \%$ in the fenofibrate group versus $6.6 \%$ in the placebo group $(\mathrm{P}=0.01$ for interaction). There was also a nonsignificant suggestion of heterogeneity when patients who had a triglyceride level in the highest third $(\geq 204 \mathrm{mg}$ per deciliter $[\geq 2.30$ mmol per liter]) and an HDL cholesterol level in the lowest third ( $\leq 34 \mathrm{mg}$ per deciliter $[\leq 0.88$ mmol per liter]) were compared with all the other patients $(\mathrm{P}=0.057$ for interaction). In this subgroup of patients with high triglyceride levels and low HDL cholesterol levels, the primary outcome rate was $12.4 \%$ in the fenofibrate group, versus $17.3 \%$ in the placebo group, whereas such rates were $10.1 \%$ in both study groups for all other patients.

\section{DISCUSSION}

In this trial, we tested the hypothesis that the use of fenofibrate to increase plasma HDL cholesterol levels and to reduce plasma triglyceride levels in patients with type 2 diabetes who were already receiving simvastatin therapy would result in an additional cardiovascular benefit, as compared with simvastatin therapy alone. However, the rates of the primary outcome did not differ significantly between the fenofibrate group and the placebo group during 4.7 years of treatment and follow-up.

When a study does not support the central hypothesis, it is critical to examine potential reasons for this outcome. One possibility is that the addition of fenofibrate to statin therapy benefited only certain subgroups of patients and that other subgroups that did not benefit diluted the overall effect. Our study was part of a factorial design to simultaneously test the effects of 
intensive glycemic control ${ }^{17,20}$ and combination lipid therapy on cardiovascular outcomes. To allow for efficient enrollment of the entire cohort of 10,000 patients while including a group for whom the results of the lipid trial could be widely extrapolated, we used broader inclusion criteria for plasma lipid levels than might have been used if the lipid trial had been an independent study.

A second possibility is that the trial might have had fewer events than anticipated. However, the annual rate of $2.4 \%$ in the placebo group was the rate used in the power calculations. Another possibility is poor adherence to the experimental protocol. However, adherence at the end of the study was approximately $80 \%$ in both the fenofibrate and placebo groups and $80 \%$ for simvastatin. Furthermore, unlike the FIELD study, in which there was a disproportionate drop-in to statin therapy in the placebo group,${ }^{11}$ the prevalence of statin therapy in our study was similar in the fenofibrate and placebo groups. A fourth possibility is that fenofibrate is not as effective as gemfibrozil, which showed benefit in the Helsinki Heart Study (HHS) and VAHIT, ${ }^{13,} 15$ studies in which there was no background statin therapy.

In examined subgroups, only sex had a significant interaction with treatment: men seemed to benefit from fenofibrate therapy, whereas there was a trend toward harm among women. This is in contrast to the results of the FIELD study, in which there was no significant interaction effect between treatment and sex on outcome. ${ }^{11}$

There was also a suggestion of heterogeneity according to baseline lipid levels: patients who had both a triglyceride level in the highest third and an HDL cholesterol level in the lowest third (which we termed the subgroup with dyslipidemia) appeared to benefit from fenofibrate, whereas all other patients receiving fenofibrate did not. The mean baseline HDL cholesterol level in the subgroup with dyslipidemia was $29.5 \mathrm{mg}$ per deciliter $(0.76 \mathrm{mmol}$ per liter), and the median triglyceride level was $284 \mathrm{mg}$ per deciliter ( $3.21 \mathrm{mmol}$ per liter), in contrast to the rest of the patients, in whom the mean HDL cholesterol level was $39.9 \mathrm{mg}$ per deciliter (1.03 mmol per liter) and the median triglyceride level was $144 \mathrm{mg}$ per deciliter $(1.63 \mathrm{mmol}$ per liter). From baseline to 4 months in the fenofibrate group, the HDL cholesterol level rose $12.9 \%$ and the triglyceride level fell $35.0 \%$ among patients in the subgroup with dyslipidemia, as compared with a $7.3 \%$ rise in the HDL cholesterol level and a $24.1 \%$ decrease in the triglyceride level among all other patients receiving fenofibrate. The treatment interaction according to sex for the entire ACCORD Lipid cohort was not observed in the subgroup with dyslipidemia (data not shown).

The results for patients in the subgroup with dyslipidemia are similar to those in post hoc subgroup analyses performed in three of four major fibrate trials, including HHS, ${ }^{24}$ the Bezafibrate Infarction Prevention (BIP) trial, ${ }^{14}$ and the FIELD trial12 (see Section 19 in Supplementary Appendix 1 for details). Our subgroup results and those of these previous trials support the view that the addition of fenofibrate to a statin may benefit patients with type 2 diabetes who have substantial dyslipidemia. The use of combination fibrate-statin therapy in such patients is consistent with current guidelines that recommend treatment for patients with hypertriglyceridemia and low HDL cholesterol levels that persist despite statin therapy. ${ }^{25}$

Previous studies ${ }^{11,22}$ have raised concern about increases in serum creatinine levels during fenofibrate treatment. Serum creatinine levels increased in the fenofibrate group soon after randomization but thereafter remained constant, as compared with those in the placebo group. In the FIELD study, there was a return of serum creatinine to baseline levels by 8 weeks after the end of the trial. ${ }^{11}$ In our study, there was no significant difference in the incidence of endstage renal disease or need for dialysis between the fenofibrate group and the placebo group. There was a reduction in both microalbuminuria and macroalbuminuria in the fenofibrate group. There has also been longstanding concern regarding an increased risk of myositis or 
rhabdomyolysis when fibrates are added to statins. ${ }^{26,27}$ No evidence for such a risk was noted in our study, a finding that was compatible with evidence that fenofibrate, in contrast to gemfibrozil, does not increase plasma concentrations of statins. ${ }^{28}$

In conclusion, we found that combination therapy with the use of fenofibrate and simvastatin (at a daily dose of $40 \mathrm{mg}$ or less) did not reduce rates of cardiovascular disease, as compared with simvastatin alone. Our findings do not support the use of combination fibrate-statin therapy, rather than statin therapy alone, to reduce cardiovascular risk in the majority of patients with type 2 diabetes who are at high risk for cardiovascular disease.

\section{Acknowledgments}

Supported by the National Heart, Lung, and Blood Institute (contracts N01-HC-95178, N01-HC-95179, N01HC-95180, N01-HC-95181, N01-HC-95182, N01-HC-95183, N01-HC-95184, IAAY1-HC-9035, and IAAY1HC-1010), the National Institute of Diabetes and Digestive and Kidney Diseases, the National Institute on Aging, the National Eye Institute, the Centers for Disease Control and Prevention, and General Clinical Research Centers at many sites. The following companies provided study medications, equipment, or supplies: Abbott Laboratories, Amylin Pharmaceutical, AstraZeneca Pharmaceuticals, Bayer HealthCare, Closer Healthcare, GlaxoSmithKline Pharmaceuticals, King Pharmaceuticals, Merck, Novartis Pharmaceuticals, Novo Nordisk, Omron Healthcare, SanofiAventis, and Takeda Pharmaceuticals.

Dr. Ginsberg reports receiving consulting fees from Merck, Merck Schering-Plough, Bristol-Myers Squibb, AstraZeneca, Abbott, Roche, Isis/Genzyme, GlaxoSmithKline, Novartis, Pfizer, and Regeneron/Sanofi-Aventis and grant support from Merck, Isis/Genzyme, Roche, and AstraZeneca; Dr. Elam, receiving consulting fees from Pfizer, Abbott, and Merck Schering-Plough; Dr. Crouse, receiving consulting fees from the National Lipid Association, AstraZeneca, Merck, and Merck Schering-Plough and grant support from AstraZeneca; Dr. Leiter, receiving consulting fees from AstraZeneca, Merck, Pfizer, Roche, and Solvay and grant support from AstraZeneca, Merck, Pfizer, and Roche; Dr. Linz, having an equity interest in Pfizer, Novartis, and AstraZeneca; Dr. Buse, receiving consulting fees from Novo Nordisk, Amylin, Becton Dickinson, Eli Lilly, Hoffmann-La Roche, GlycoMark, Wyeth, Daiichi Sankyo, Bristol-Myers Squibb, Bayhill Therapeutics, LipoScience, MannKind, Veritas, MicroIslet, GlaxoSmithKline, Abbott, Exsulin, and GI Dynamics and grant support from Amylin, Novo Nordisk, Medtronic, Eli Lilly, Novartis, Tolerex, Osiris, Halozyme, Pfizer, Hoffmann-La Roche, InterKrin, Merck, Sanofi-Aventis, Dexcom, Johnson \& Johnson, Bristol-Myers Squibb, and Fujisawa, having an equity interest in Insulet, and providing expert testimony for Novo Nordisk; Dr. Gerstein, receiving consulting fees from Sanofi-Aventis, GlaxoSmithKline, Eli Lilly, Novo Nordisk, AstraZeneca, Bristol-Myers Squibb, Roche, Medtronic, Merck, Bayer, Bioavail, and Janssen-Ortho, grant support from Sanofi-Aventis, GlaxoSmithKline, Novo Nordisk, Merck, Pronova, and Roche, and lecture fees from SanofiAventis, GlaxoSmithKline, Solvay, Boehringer Ingelheim, Servier, Bayer, Eli Lilly, Novo Nordisk, and Takeda; Dr. Probstfield, receiving grant support from Sanofi-Aventis, Boehringer Ingelheim, and Abbott; Dr. Grimm, receiving consulting and lecture fees and grant support from Pfizer, Merck, and Novartis, consulting and lecture fees from Takeda, and lecture fees from AstraZeneca, Forest Laboratories, and Schering-Plough; Dr. Bigger, receiving consulting fees from Merck and Roche; Dr. Goff, receiving consulting fees from Takeda and grant support from Merck; and Dr. Cushman, receiving consulting fees from Novartis, Takeda, Sanofi-Aventis, Bristol-Myers Squibb, King, Daiichi-Sankyo, Gilead, Theravance, Pharmacopeia, and Sciele and grant support from Novartis, GlaxoSmithKline, and Merck. No other potential conflict of interest relevant to this article was reported.

\section{APPENDIX}

The affiliations of the members of the writing committee are as follows: the Department of Medicine, Columbia University College of Physicians and Surgeons, New York (H.N.G.); Memphis Veterans Affairs Medical Center, Memphis (M.B.E., W.C.C.); the Department of Public Health Sciences (L.C.L., D.C.G., R.P.B.) and Preventive Cardiology Program (J.R.C.), Wake Forest University School of Medicine, Winston-Salem, NC; University of Toronto, Toronto (L.A.L.); Naval Medical Center, San Diego, CA (P.L.); the Departments of Biostatistics and Epidemiology, Columbia University Mailman School of Public Health, New York (W.T.F.); the Division of Endocrinology, University of North Carolina School of Medicine, Chapel Hill (J.B.B.); the Department of Medicine and the Population Health Research Institute, McMaster University, Hamilton, ON, Canada (H.C.G.); the University of Washington, Seattle (J.P.); the Berman Center for Outcomes and Clinical Research, Minneapolis (R.H.G.), the Departments of Medicine and Physiology and Biophysics, Case 
Western Reserve University, Cleveland (F.I.-B.); the Division of Cardiology, Columbia University College of Physicians and Surgeons, New York (J.T.B.); and the National Heart, Lung, and Blood Institute, Bethesda, MD (D.G.S.-M.).

\section{REFERENCES}

1. Almdal T, Scharling H, Jensen JS, Vestergaard H. The independent effect of type 2 diabetes mellitus on ischemic heart disease, stroke, and death: a population-based study of 13,000 men and women with 20 years of follow-up. Arch Intern Med 2004;164:1422-1426. [PubMed: 15249351]

2. Stamler J, Vaccaro O, Neaton JD, Wentworth D. Diabetes, other risk factors, and 12-yr cardiovascular mortality for men screened in the Multiple Risk Factor Intervention Trial. Diabetes Care 1993;16:434444. [PubMed: 8432214]

3. Haffner SM, Lehto S, Rönnemaa T, Pyörälä K, Laakso M. Mortality from coronary heart disease in subjects with type 2 diabetes and in nondiabetic subjects with and without prior myocardial infarction. N Engl J Med 1998;339:229-234. [PubMed: 9673301]

4. Miettinen H, Lehto S, Salomaa V, et al. Impact of diabetes on mortality after the first myocardial infarction. Diabetes Care 1998;21:69-75. [PubMed: 9538972]

5. Chahil TJ, Ginsberg HN. Diabetic dyslipidemia. Endocrinol Metab Clin North Am 2006;35:491-510. [PubMed: 16959582]

6. Turner RC, Millns H, Neil HA, et al. Risk factors for coronary artery disease in non-insulin dependent diabetes mellitus: United Kingdom Prospective Diabetes Study (UKPDS: 23). BMJ 1998;316:823828. [PubMed: 9549452]

7. Collins R, Armitage J, Parish S, Sleigh P, Peto R. MRC/BHF Heart Protection Study of cholesterollowering with simvastatin in 5963 people with diabetes: a randomised placebo-controlled trial. Lancet 2003;361:2005-2016. [PubMed: 12814710]

8. Shepherd J, Barter P, Carmena R, et al. Effect of lowering LDL cholesterol substantially below currently recommended levels in patients with coronary heart disease and diabetes: the Treating to New Targets (TNT) study. Diabetes Care 2006;29:1220-1226. [PubMed: 16731999]

9. Colhoun HM, Betteridge DJ, Durrington PN, et al. Primary prevention of cardiovascular disease with atorvastatin in type 2 diabetes in the Collaborative Atorvastatin Diabetes Study (CARDS): multicentre randomised placebo-controlled trial. Lancet 2004;364:685-696. [PubMed: 15325833]

10. Rubins HB, Robins SJ, Collins D, et al. Diabetes, plasma insulin, and cardiovascular disease: subgroup analysis from the Department of Veterans Affairs high-density lipoprotein intervention trial (VAHIT). Arch Intern Med 2002;162:2597-2604. [PubMed: 12456232]

11. Keech A, Simes RJ, Barter P, et al. Effects of long-term fenofibrate therapy on cardiovascular events in 9795 people with type 2 diabetes mellitus (the FIELD study): randomised controlled trial. Lancet 2005;366:1849-1861. [Errata, Lancet 2006;368:1415, 1420.]. [PubMed: 16310551]

12. Scott R, O'Brien R, Fulcher G, et al. Effects of fenofibrate treatment on cardiovascular disease risk in 9,795 individuals with type 2 diabetes and various components of the metabolic syndrome: the Fenofibrate Intervention and Event Lowering in Diabetes (FIELD) study. Diabetes Care 2009;32:493-498. [PubMed: 18984774]

13. Frick MH, Elo O, Haapa K, et al. Helsinki Heart Study: primary-prevention trial with gemfibrozil in middle-aged men with dyslipidemia.Safety of treatment, changes in risk factors, and incidence of coronary heart disease. N Engl J Med 1987;317:1237-1245. [PubMed: 3313041]

14. Secondary prevention by raising HDL cholesterol and reducing triglycerides in patients with coronary artery disease: the Bezafibrate Infarction Prevention (BIP) study. Circulation 2000;102:21-27. [PubMed: 10880410]

15. Rubins HB, Robins SJ, Collins D, et al. Gemfibrozil for the secondary prevention of coronary heart disease in men with low levels of high-density lipoprotein cholesterol. N Engl J Med 1999;341:410418. [PubMed: 10438259]

16. Buse JB, Bigger JT, Byington RP, et al. Action to Control Cardiovascular Risk in Diabetes (ACCORD) trial: design and methods. Am J Cardiol 2007;99:21i-33i. 
17. Goff DC Jr, Gerstein HC, Ginsberg HN, et al. Prevention of cardiovascular disease in persons with type 2 diabetes mellitus: current knowledge and rationale for the Action to Control Cardiovascular Risk in Diabetes (ACCORD) trial. Am J Cardiol 2007;99:4i-20i.

18. Ginsberg HN, Bonds DE, Lovato LC, et al. Evolution of the lipid trial protocol of the Action to Control Cardiovascular Risk in Diabetes (ACCORD) trial. Am J Cardiol 2007;99:56i-67i.

19. Cushman WC, Grimm RH Jr, Cutler JA, et al. Rationale and design for the blood pressure intervention of the Action to Control Cardiovascular Risk in Diabetes (ACCORD) trial. Am J Cardiol 2007;99:44i-55i.

20. The Action to Control Cardiovascular Risk in Diabetes Study Group. Effects of intensive glucose lowering in type 2 diabetes. N Engl J Med 2008;358:2545-2559. [PubMed: 18539917]

21. Executive Summary of the Third Report of the National Cholesterol Education Program (NCEP) Expert Panel on Detection, Evaluation, and Treatment of High Blood Cholesterol in Adults (Adult Treatment Panel III). JAMA 2001;285:2486-2497. [PubMed: 11368702]

22. Genest J, Frohlich J, Steiner G. Effect of fenofibrate-mediated increase in plasma homocysteine on the progression of coronary artery disease in type 2 diabetes mellitus. Am J Cardiol 2004;93:848853. [PubMed: 15050487]

23. Levey AS, Bosch JP, Lewis JB, Greene T, Rogers N, Roth D. A more accurate method to estimate glomerular filtration rate from serum creatinine: a new prediction equation. Ann Intern Med 1999;130:461-470. [PubMed: 10075613]

24. Manninen V, Tenkanen L, Koskinen P, et al. Joint effects of serum triglyceride and LDL cholesterol and HDL cholesterol concentrations on coronary heart disease risk in the Helsinki Heart Study: implications for treatment. Circulation 1992;85:37-45. [PubMed: 1728471]

25. Grundy SM, Cleeman JI, Merz CN, et al. Implications of recent clinical trials for the National Cholesterol Education Program Adult Treatment Panel III guidelines. Circulation 2004;110:227239. [Erratum, Circulation 2004;110:763.]. [PubMed: 15249516]

26. Pasternak RC, Smith SC Jr, BaireyMerz CN, Grundy SM, Cleeman JI, Lenfant C. ACC/AHA/NHLBI clinical advisory on the use and safety of statins. Circulation 2002;106:1024-1028. [PubMed: 12186811]

27. Jones PH, Davidson MH. Reporting rate of rhabdomyolysis with fenofibrate + statin versus gemfibrozil + any statin. Am J Cardiol 2005;95:120-122. [PubMed: 15619408]

28. Bergman AJ, Murphy G, Burke J, et al. Simvastatin does not have a clinically significant pharmacokinetic interaction with fenofibrate in humans. J Clin Pharmacol 2004;44:1054-1062. [PubMed: 15317833] 


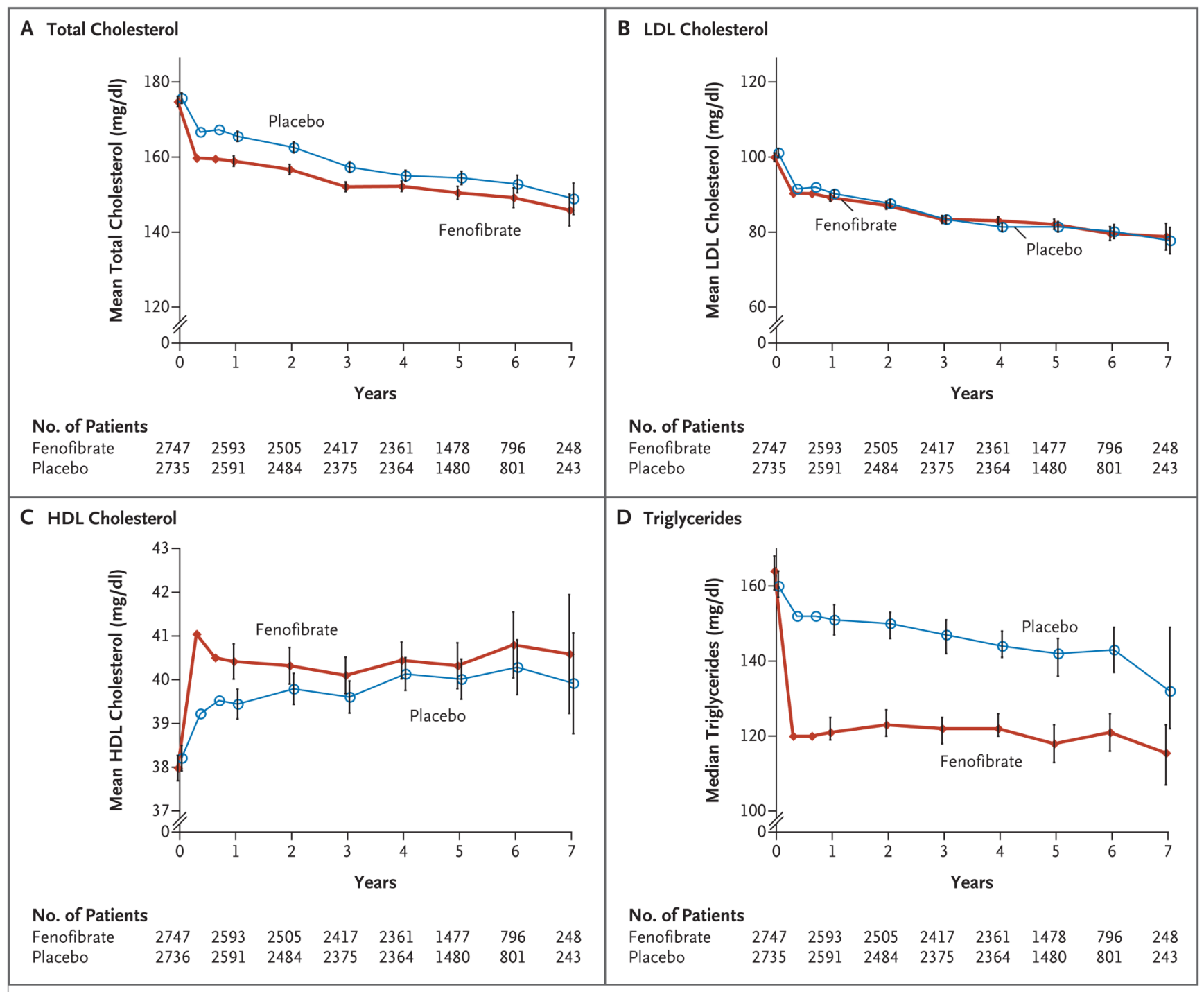

Figure 1. Lipid Values

Shown are mean plasma levels of total cholesterol (Panel A), low-density lipoprotein (LDL) cholesterol (Panel B), and high-density lipoprotein (HDL) cholesterol (Panel C) and median levels of triglycerides (Panel D) at baseline, 4 months, 8 months, 1 year, and annually thereafter. Nominal $\mathrm{P}$ values for differences between the study groups at 4 months and at the end of the study were, respectively: total cholesterol, $\mathrm{P}<0.001$ and $\mathrm{P}=0.02 ; \mathrm{LDL}$ cholesterol, $\mathrm{P}=0.11$ and $\mathrm{P}=0.16$; HDL cholesterol, $\mathrm{P}<0.001$ and $\mathrm{P}=0.01$; and triglycerides, $\mathrm{P}<0.001$ for both comparisons with the use of nonparametric tests. End-of-study visits were those that occurred in early 2009 and included follow-up at years 4, 5, 6, and 7. The I bars represent $95 \%$ confidence intervals. To convert the values for cholesterol to millimoles per liter, multiply by 0.02586 . To convert the values for triglycerides to millimoles per liter, multiply by 0.01129 . 


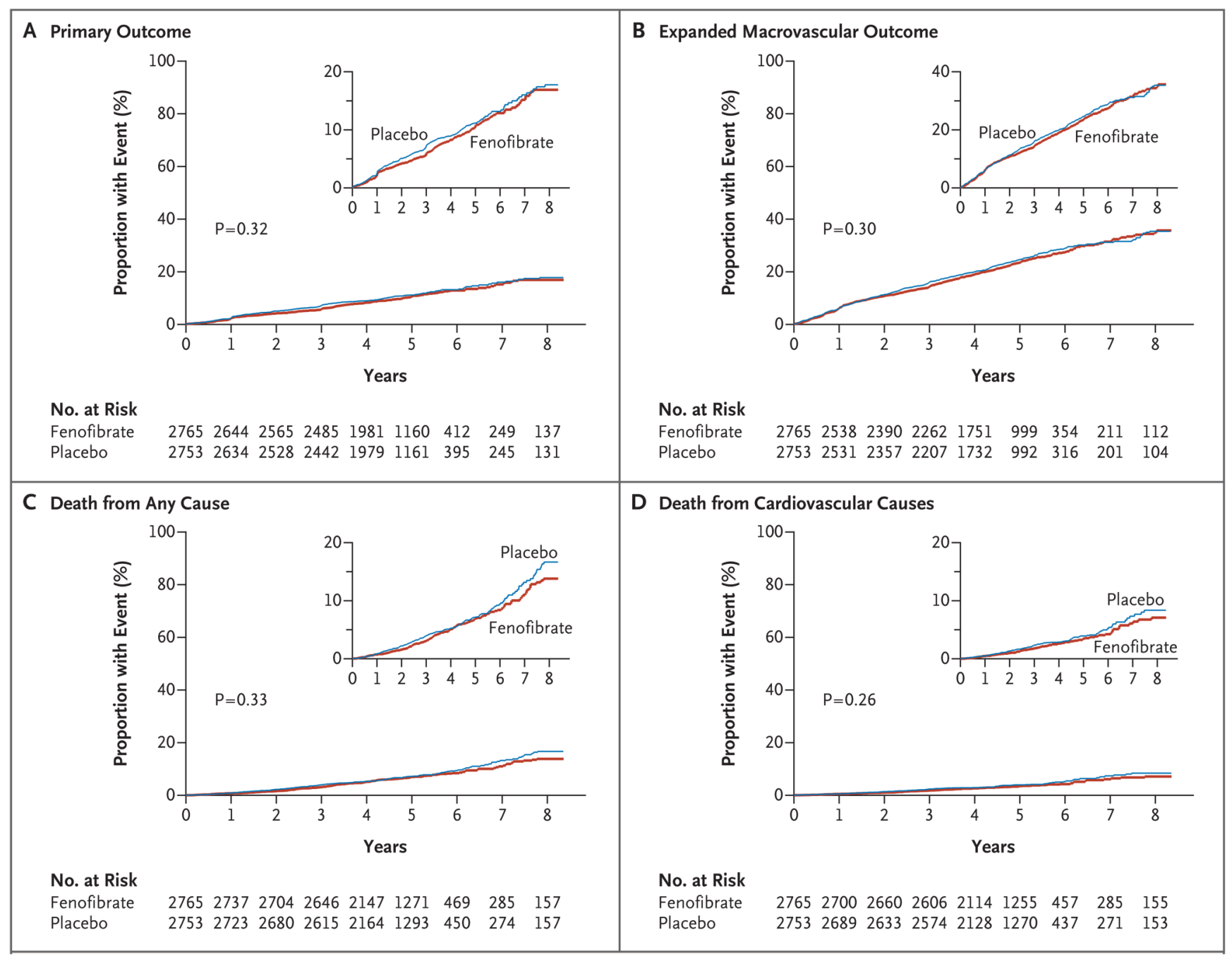

Figure 2. Kaplan-Meier Analyses of the Primary Outcome, Expanded Macrovascular Outcome, and Death

Shown are the cumulative incidence of the primary outcome (nonfatal myocardial infarction, nonfatal stroke, or death from cardiovascular causes) (Panel A), the expanded macrovascular outcome (a combination of the primary outcome plus revascularization or hospitalization for congestive heart failure) (Panel B), and death from any cause (Panel C) or from cardiovascular causes (Panel D) during follow-up. The insets show close-up versions of the graphs in each panel. 


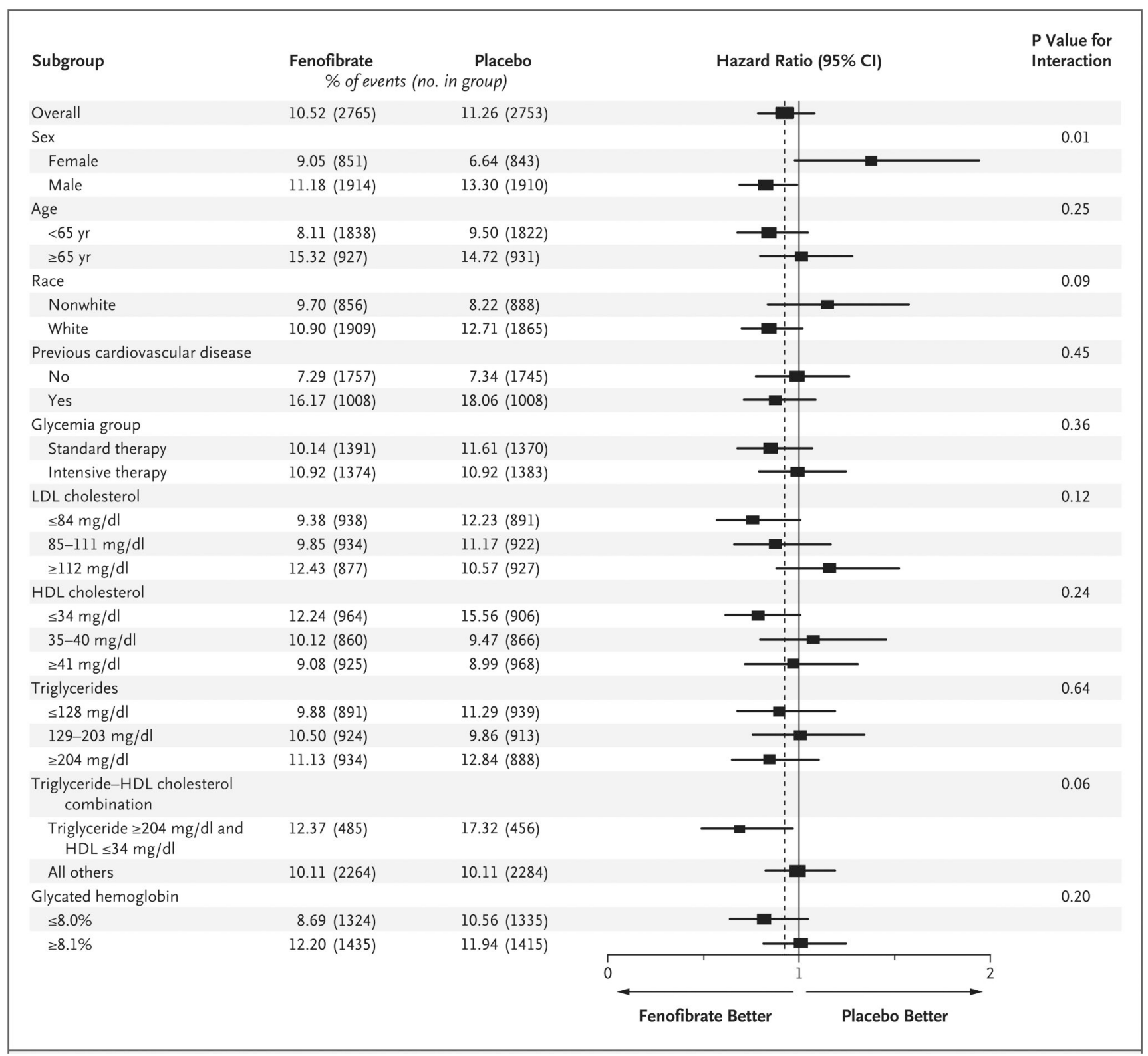

Figure 3. Hazard Ratios for the Primary Outcome in Prespecified Subgroups

The horizontal bars represent $95 \%$ confidence intervals, and the vertical dashed line indicates the overall hazard ratio. The size of each square is proportional to the number of patients. $\mathrm{P}$ values are for tests for interaction. To convert the values for cholesterol to millimoles per liter, multiply by 0.02586 . To convert the values for triglycerides to millimoles per liter, multiply by 0.01129 . 


\section{Table 1}

Baseline Characteristics of the Patients. ${ }^{*}$

\begin{tabular}{|c|c|c|c|c|}
\hline Characteristic & $\begin{array}{r}\text { All Patients } \\
(\mathbf{N}=\mathbf{5 5 1 8})\end{array}$ & $\begin{array}{r}\text { Fenofibrate } \\
(N=2765)\end{array}$ & $\begin{array}{r}\text { Placebo } \\
(\mathrm{N}=\mathbf{2 7 5 3})\end{array}$ & P Value \\
\hline Age $-\mathrm{yr}$ & $62.3 \pm 6.8$ & $62.2 \pm 6.7$ & $62.3 \pm 6.9$ & 0.69 \\
\hline Female sex - no. $(\%)$ & $1694(30.7)$ & $851(30.8)$ & $843(30.6)$ & 0.90 \\
\hline \multicolumn{5}{|l|}{ Race or ethnic group - no. $(\%)^{\dagger}$} \\
\hline White & $3774(68.4)$ & $1909(69.0)$ & $1865(67.7)$ & 0.30 \\
\hline Black & $834(15.1)$ & $392(14.2)$ & $442(16.1)$ & 0.05 \\
\hline Hispanic & $407(7.4)$ & $213(7.7)$ & $194(7.0)$ & 0.35 \\
\hline Education — no. $(\%)$ & & & & 0.19 \\
\hline Less than high school & $750(13.6)$ & $394(14.2)$ & $356(12.9)$ & \\
\hline High-school graduate or GED & $1433(26.0)$ & $712(25.8)$ & $721(26.2)$ & \\
\hline Some college & $1827(33.1)$ & $885(32.0)$ & $942(34.2)$ & \\
\hline College degree or higher & $1505(27.3)$ & $772(27.9)$ & $733(26.6)$ & \\
\hline Missing data & $3(<0.1)$ & $2(0.1)$ & $1(<0.1)$ & \\
\hline Previous cardiovascular event - no. $(\%)$ & $2016(36.5)$ & $1008(36.5)$ & $1008(36.6)$ & 0.90 \\
\hline Previous congestive heart failure - no. $(\%)$ & $291(5.3)$ & $151(5.5)$ & $140(5.1)$ & 0.54 \\
\hline Cigarette-smoking status - no. $(\%)$ & & & & 0.42 \\
\hline Current & $803(14.6)$ & $410(14.8)$ & $393(14.3)$ & \\
\hline Former & $2546(46.2)$ & $1292(46.7)$ & $1254(45.6)$ & \\
\hline Never & $2161(39.2)$ & $1059(38.3)$ & $1102(40.0)$ & \\
\hline Missing data & $8(0.1)$ & $4(0.1)$ & $4(0.1)$ & \\
\hline Weight $-\mathrm{kg}$ & $94.8 \pm 18.7$ & $94.5 \pm 18.5$ & $95.2 \pm 18.8$ & 0.21 \\
\hline Body-mass index & $32.3 \pm 5.4$ & $32.2 \pm 5.4$ & $32.4 \pm 5.4$ & 0.32 \\
\hline \multicolumn{5}{|l|}{ Blood pressure $-\mathrm{mm} \mathrm{Hg}$} \\
\hline Systolic & $133.9 \pm 17.8$ & $133.8 \pm 17.7$ & $134.0 \pm 17.9$ & 0.79 \\
\hline Diastolic & $74.0 \pm 10.8$ & $73.9 \pm 10.7$ & $74.0 \pm 10.9$ & 0.58 \\
\hline \multicolumn{5}{|l|}{ Medications - no. $(\%)$} \\
\hline Insulin & $1836(33.3)$ & $919(33.2)$ & $917(33.3)$ & 0.95 \\
\hline Metformin & $3420(62.0)$ & $1712(61.9)$ & $1708(62.0)$ & 0.92 \\
\hline Any sulfonylurea & $2892(52.4)$ & $1440(52.1)$ & $1452(52.7)$ & 0.62 \\
\hline Any thiazolidinedione & 973 (17.6) & $480(17.4)$ & $493(17.9)$ & 0.59 \\
\hline Angiotensin-converting-enzyme inhibitor & $2967(53.8)$ & $1473(53.3)$ & $1494(54.3)$ & 0.46 \\
\hline Angiotensin-receptor blocker & $838(15.2)$ & $405(14.6)$ & $433(15.7)$ & 0.26 \\
\hline Aspirin & $3106(56.3)$ & $1583(57.3)$ & $1523(55.3)$ & 0.15 \\
\hline Beta-blocker & $1798(32.6)$ & $912(33.0)$ & $886(32.2)$ & 0.53 \\
\hline Any thiazide diuretic & $1473(26.7)$ & $740(26.8)$ & $733(26.6)$ & 0.91 \\
\hline Statin & $3299(59.8)$ & $1641(59.3)$ & $1658(60.2)$ & 0.51 \\
\hline Any lipid-lowering agent & $3558(64.5)$ & $1773(64.1)$ & $1785(64.8)$ & 0.58 \\
\hline
\end{tabular}

N Engl J Med. Author manuscript; available in PMC 2010 October 29. 


\begin{tabular}{|c|c|c|c|c|}
\hline Characteristic & $\begin{array}{r}\text { All Patients } \\
(\mathbf{N}=5518)\end{array}$ & $\begin{array}{r}\text { Fenofibrate } \\
(\mathbf{N}=\mathbf{2 7 6 5})\end{array}$ & $\begin{array}{r}\text { Placebo } \\
(\mathbf{N}=\mathbf{2 7 5 3})\end{array}$ & P Value \\
\hline \multicolumn{5}{|l|}{ Duration of diabetes $-\mathrm{yr}$} \\
\hline Median & 9 & 10 & 9 & 0.83 \\
\hline Interquartile range & $5-15$ & $5-15$ & $5-15$ & \\
\hline \multicolumn{5}{|l|}{ Glycated hemoglobin - \% } \\
\hline Mean & $8.3 \pm 1.0$ & $8.3 \pm 1.0$ & $8.3 \pm 1.0$ & 0.52 \\
\hline Median & 8.1 & 8.1 & 8.1 & \\
\hline Interquartile range & $7.6-8.8$ & $7.6-8.8$ & $7.5-8.8$ & \\
\hline Fasting plasma glucose $-\mathrm{mg} / \mathrm{dl}$ & $175.8 \pm 54.9$ & $176.5 \pm 54.5$ & $175.1 \pm 55.3$ & 0.38 \\
\hline Amputation due to diabetes - no. $(\%)$ & $110(2.0)$ & $59(2.1)$ & $51(1.9)$ & 0.45 \\
\hline Potassium $-\mathrm{mg} / \mathrm{dl}$ & $4.5 \pm 0.4$ & $4.5 \pm 0.4$ & $4.5 \pm 0.4$ & 0.31 \\
\hline Serum creatinine $-\mathrm{mg} / \mathrm{dl}$ & $0.9 \pm 0.2$ & $0.9 \pm 0.2$ & $0.9 \pm 0.2$ & 0.96 \\
\hline \multicolumn{5}{|c|}{ Estimated glomerular filtration rate — no. (\%) } \\
\hline $30-49 \mathrm{ml} / \mathrm{min} / 1.73 \mathrm{~m}^{2}$ & $141(2.6)$ & $71(2.6)$ & $70(2.5)$ & 0.89 \\
\hline$>50 \mathrm{ml} / \mathrm{min} / 1.73 \mathrm{~m}^{2}$ & $5347(97.4)$ & $2668(97.4)$ & $2679(97.5)$ & \\
\hline \multicolumn{5}{|l|}{ Plasma cholesterol $-\mathrm{mg} / \mathrm{dl}$} \\
\hline Total & $175.2 \pm 37.3$ & $174.7 \pm 36.8$ & $175.7 \pm 37.9$ & 0.36 \\
\hline Low-density lipoprotein & $100.6 \pm 30.7$ & $100.0 \pm 30.3$ & $101.1 \pm 31.0$ & 0.15 \\
\hline High-density lipoprotein & $38.1 \pm 7.8$ & $38.0 \pm 7.8$ & $38.2 \pm 7.8$ & 0.25 \\
\hline \multicolumn{5}{|l|}{ Plasma triglyceride $-\mathrm{mg} / \mathrm{dl}$} \\
\hline Median & 162 & 164 & 160 & 0.15 \\
\hline Interquartile range & $113-229$ & $114-232$ & $112-227$ & \\
\hline
\end{tabular}

* Plus-minus values are means \pm SD. Percentages may not total 100 because of rounding. To convert the values for glucose to millimoles per liter, multiply by 0.055551 . To convert the values for cholesterol to millimoles per liter, multiply by 0.02586 . To convert the values for triglycerides to millimoles per liter, multiply by 0.01129 . To convert the values for potassium to millimoles per liter, multiply by 0.2558 . To convert the values for creatinine to micromoles per liter, multiply by 88.4 . GED denotes general equivalency diploma.

${ }^{\dagger}$ Race or ethnic group was self-reported, and patients could check multiple categories.

${ }^{*}$ The body-mass index is the weight in kilograms divided by the square of the height in meters. 


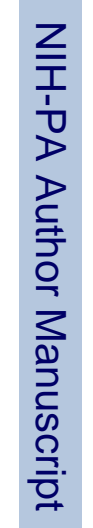

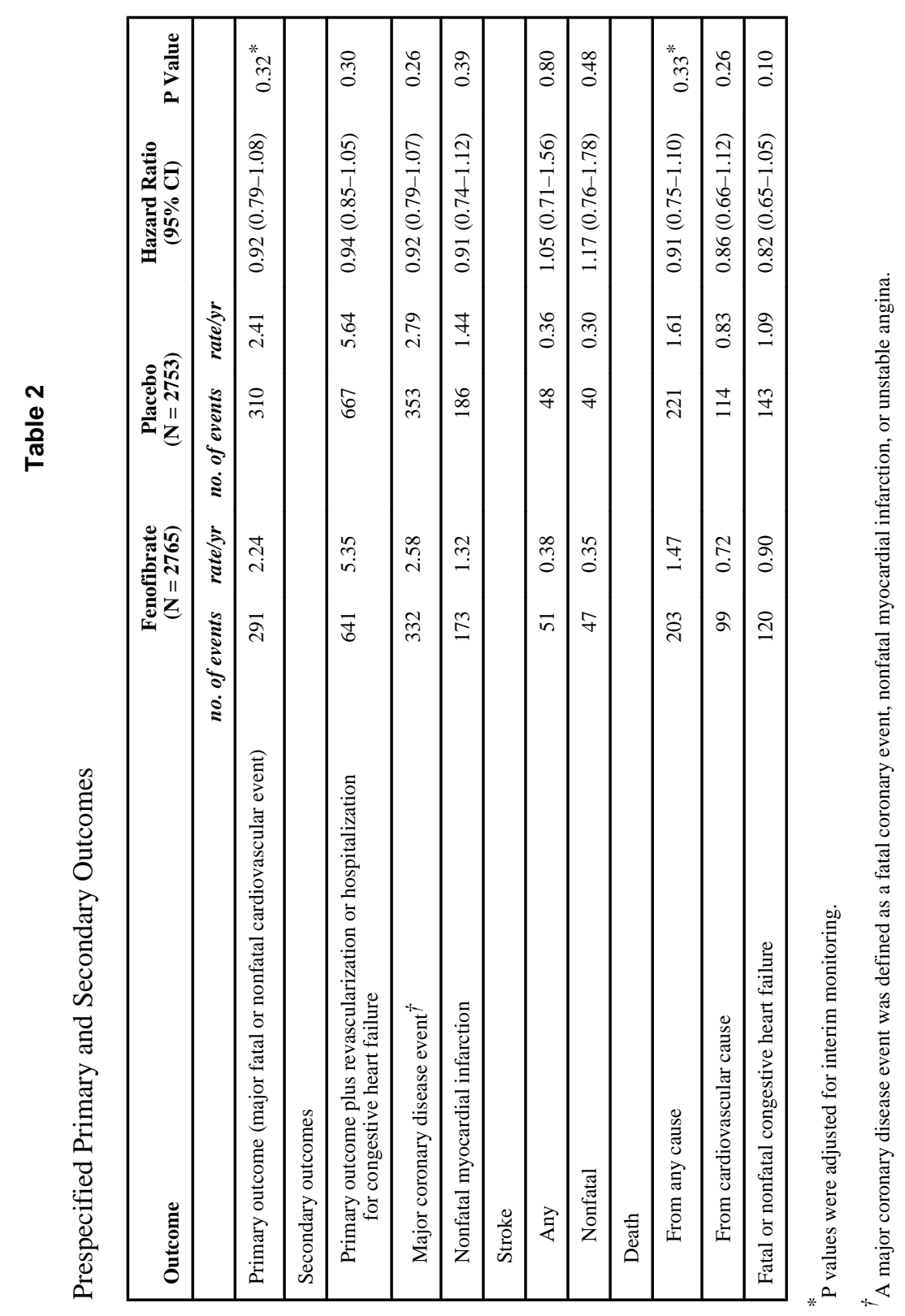

N Engl J Med. Author manuscript; available in PMC 2010 October 29. 